Original article

\title{
The costs and benefits of pressure injury point prevalence auditing
}

\author{
Floyd E, Hodgkins R, Naylon L, Noon M, Sirotti L and Monaro S
}

Keywords pressure injury, point prevalence, auditing, costs, benefits

For referencing Floyd E et al. The costs and benefits of pressure injury point prevalence auditing. Wound Practice and Research 2020; 28(2):66-74.

DOI https://doi.org/10.33235/wpr.28.2.66-74

\section{Emma Floyd}

$\mathrm{RN}, \mathrm{BN}, \mathrm{MN}$

Aged Care Rapid Response CNC

Northern Sydney Local Health District, NSW

Rachel Hodgkins

RN, BSc (Hons) Nursing, Grad Cert Stomal Therapy,

Grad Cert Clinical Redesign

Wound and Stomal Therapy CNC

Royal North Shore Hospital, NSW

\section{Lisa Naylon}

RN, BN, Grad Cert Stomal Therapy,

Grad Cert Ophthalmology Nursing

Wound and Stomal Therapy CNS2, Ryde Hospital, NSW

\section{Michelle Noon}

RN, BN, Grad Dip Wound Management

Wound and Stomal Therapy CNC, Hornsby Hospital,

NSW

\section{Larissa Sirotti}

RN, BN, MN, Grad Cert Intensive Care, Grad Cert

Clinical Management

CNS Intensive Care Unit

Royal North Shore Hospital, NSW

\section{Susan Monaro*}

RN, BAppSc (Nursing), MN, PhD

Skin Integrity Improvement Facilitator

Clinical Governance Unit

Northern Sydney Local Health District, NSW

Clinical Senior Lecturer

Susan Wakil School of Nursing and Midwifery

University of Sydney, NSW

Email sue.monaro@health.nsw.gov.au

\section{Abstract}

Pressure injuries remain a focus for patient safety and quality initiatives, particularly in acute care settings. This focus has been facilitated by the generation of data on pressure injury point prevalence which began publication in Australia in 2003. Since then, many healthcare organisations have undertaken serial pressure injury point prevalence audits (PIPPA) as part of a comprehensive program to measure and address pressure injuries. In our local health district, there has been a commitment to an annual PIPPA, but recently we sought to determine if we had reached a tipping point where the relative benefits of collecting this snapshot data may no longer justify the costs, particularly given the economic environment in the Australian healthcare system. The annual cost of conducting a district-wide PIPPA was calculated, and this is discussed alongside both non-financial costs and organisational and auditor benefits.

\section{Key points}

What is already known about this topic

- Pressure injuries are a significant burden to the person and the healthcare system.

- Pressure injury point prevalence audits (PIPPA) provide trended data.

What this manuscript contributes

- Costs associated with PIPPA.

- Benefits associated with PIPPA.

\section{Introduction}

Wounds are receiving increased attention in terms of both prevention and management with a need to more clearly define the scope of the problem. Initiatives to address wound prevention and management have become a priority for health services. Wounds include pressure injuries as well as skin tears, leg ulcers and moisture-associated skin damage. However, many of these can be misclassified as pressure 
injuries, making the contribution of this type of wound difficult to cost accurately.

The management of chronic wounds in Australia has been calculated to cost US\$1.65 billion per annum ${ }^{1}$. Pressure injuries contribute to the growing burden of chronic wounds as many pressure injuries occur in patients with multiple comorbidities which trigger wound chronicity. Pressure injuries have been widely reported in the literature in terms of patient outcomes or costs and their burden on the healthcare system. Patient costs include reduced quality of life for the person and financial costs for dressings and specialised equipment. In addition, there are the vicarious emotional and financial costs to carers contributing to carer stress. Economic costs to healthcare system have been difficult to generate, but one study estimated the annual costs to the Australian healthcare system in $2012-13$ at $A \$ 983$ million which is around $1.9 \%$ of all public hospital expenditure ${ }^{2}$. In addition, opportunity costs are significant as pressure injuries increase hospital length of stay resulting in bed block and reduced capacity to admit other patients requiring inpatient care. The opportunity cost has also been provided by Nguyen, Chaboyer and Whitty ${ }^{2}$ as being in the vicinity of $\mathrm{A} \$ 820$ million per annum for 121,645 cases of pressure injuries in 2012-13, with 524,661 bed days lost. Costs also continue after an episode of inpatient care. Most commonly, post-discharge care is provided by community nursing services that have competing demands and limitations.

Healthcare organisations have contributed to raising the profile of pressure injuries and providing frameworks to systematically address the underlying issues. One initiative that has given pressure injuries a high profile was the initial publication of the Australian national safety and quality in health services standards ${ }^{3}$, where Standard 8 was dedicated to pressure injuries. The second edition incorporated pressure injuries under the comprehensive care standard ${ }^{4}$, with concerns about their reduced visibility.

International clinical practice guidelines for the prevention and treatment of pressure injuries have provided robust evidence-based recommendations for the development of frameworks by healthcare organisations. The release of the national pressure injury guidelines ${ }^{5}$ and the subsequent International guidelines in $2014^{6}$ and their revision in $2019^{7}$ have provided a very comprehensive approach to pressure injury prevention and management.

In our health district, practice guidelines have been available since 2008, prior to the national release in 2012, in the Pressure injury prevention program policy and these have been updated as new evidence emerged ${ }^{8}$. The New South Wales health policy PD2014-0079 provided further requirements for pressure injury prevention and management in the public health system including mandatory reporting and auditing. The introduction of the hospital-acquired complications (HAC) toolkit ${ }^{10}$ has further galvanised pressure injuries as a system issues for healthcare organisations. Aligned with 'never events' from the American healthcare system, HACs provide a measure of performance and incentivised funding to avoid hospital-acquired pressure injuries (HAPIs). Pressure injuries (Stage 3 and 4, suspected deep tissue injury and unstageable) that are coded as a HAC will attract penalties for healthcare services. At this stage, a Stage $3 \mathrm{HAPI}$ will incur $A \$ 30,000$ and Stage $4 \mathrm{~A} \$ 50,000$ as a penalty, acting as an incentive for facilities to improve performance for both skin assessment on entry and pressure injury prevention.

\section{Background}

Research to uncover the scope of the pressure injury problem in the Australian setting began with the work of Prentice et al. in Victoria ${ }^{11}$. Point prevalence (the number of individuals with a pressure injury at a specific point in time ${ }^{7}$ was determined to be a useful and reproducible measure of the scope of the problem. Prentice developed a robust audit (data collection) methodology for pressure injury point prevalence audits (PIPPA). Their methodology generated data about the prevalence of pressure injuries and has been adopted across many settings in the Australian healthcare system, including WoundsWest who generated state-wide datasets in Western Australia ${ }^{12}$. An indication of the pressure injury problem globally is provided by a systematic review which found point prevalence rates in acute care settings of between $6-18.5 \%{ }^{13}$. The wide range may be related to methodological inconsistencies and mandates that if prevalence is to be used as an indicator of care, there must be standardisation of auditing processes ${ }^{7}$.

In New South Wales, the Northern Sydney Local Health District (NSLHD) has used this methodology to collect continuous data on pressure injuries since 2013. Both the auditor training resources and the Access database that were developed by WoundsWest have been used with permission in NSLHD to ensure standardised processes for data collection and management. Training resources included a PowerPoint for face-to-face auditor training and testing in pressure injury identification and staging. Access databases consist of a file or set of files that contains all the data and application features such as data entry forms. The NSLHD pressure injury point prevalence database was customised and listed all wards and units audited. This enabled the generation of reports down to ward or unit level. This database also had the capability to enter data on all wound types and provided a snapshot of the scope of all wounds across the local health district (LHD). This expanded audit was done in several of the datasets (2015 and 2017), but more commonly the audit focused on pressure injuries.

Unfortunately, this capability was lost when NSLHD decided to transition to the state-wide data management platform Quality assurances reporting system (QARS) in 2018 which allowed for the inclusion of incontinence-associated dermatitis (IAD), yet broader wound aetiologies were unable to be captured. Despite auditors across NSLHD being 
assessed as competent in classifying skin injuries to ensure accuracy in identification, it is suspected that IAD may have previously been misclassified as pressure injuries in some instances. However, one significant benefit of annual PIPPAs is that not only do they provide data on point prevalence, but they can also measure many of the other criteria specified in the national standards surrounding pressure injuries. These activities not only enrich the understanding of the problem but embed a culture of prevention and quality improvement through education, resources and equipment.

NSLHD has ensured a coordinated approach to auditing through the Clinical auditing working party which reports to the Skin integrity steering committee. The working party consists of the lead wound clinician from each facility who liaises with their respective executive management team to ensure the optimal scheduling of each sites' audit considering local events and staffing. PIPPAs are conducted annually, and auditor numbers are proportional to the bed numbers of each hospital. Training of new auditors is completed in the month leading up to the audit and involves approximately 1 hour of face-to-face or 30 minutes of online training. Skin injury classification competency testing is only available in the former, so this is the preferred training modality. May is the month allocated to PIPPA, with one hospital audited on one day of each week in May. This rolling schedule of audits allows for lead clinicians at each site to assist with audits across the LHD, ensuring that there is a high level of audit and clinical experience to maintain standardisation of PIPPAs.

However, in addition to data reliability issues that were mostly addressed through training and competency assessments, the time-consuming nature of auditing due to the requirement of a head-to-toe skin inspection led to the need to consider the time and cost of collecting point prevalence data. While there is no doubt that PIPPA has many benefits, there is a lack of appreciation of the financial and opportunity costs of PIPPA costs to the healthcare system in Australia. This paper reports our serial point prevalance data and uses records of auditor training and audit participation to calculate approximate financial costs of PIPPA. An overview of other costs and benefits is derived from qualitative data collected in auditor questionnaires.

\section{Methods}

Design

PIPPA began as a quality improvement activity in 2013 using the audit methodology described. In 2016 it became a dataset as part of a mixed methods study to evaluate a model of care for wound prevention and management. In the lead-up to the audit, the lead clinician recruited auditors and provided standardised face-to-face training to new auditors. This training covered the audit protocol and identification and classification of skin injuries. Competency assessment in pressure injury staging using clinical photographs was done at the completion of auditor training. Auditors, both new and recurrent, were confirmed and the site lead allocated a team to each ward within the facility. The allocation was done to ensure that less experienced auditors were mentored by clinicians with pressure injury staging and auditing expertise. Initially, audits were paper-based but in 2018 teams successfully transitioned to a paperless audit. On the day of the audit, the team convened for a final briefing and collection of audit resources including, more recently, a monitor on wheels (MOWs). A standardised audit tool guided data collection relating to pressure injury severity and acquisition, documentation in the medical record pertaining to elements of a pressure injury prevention and/or management plan, and an environmental survey to capture pressure redistribution equipment insitu.

\section{Sample and setting}

The profiles of the five facilities in NSLHD were of variable peer group and had a range of sizes and case mix. The facilities are identified by code and detailed in Table 1.

\section{Data collection}

Audit teams approached all patients who met inclusion criteria and performed a full skin inspection noting any abnormal findings. Other data were collected relating to risk assessment, pressure injury prevention planning (equipment, repositioning, patient education), and documentation (pressure injury prevention and/or management plan, skin assessment and incident notification).

Estimated time to complete the audit at each facility and the number of auditors who participated in training and audit were recorded. This enabled the calculation of an approximate cost of training and auditing. Lastly, we collected qualitative data via an auditor questionnaire to obtain feedback about the experience for the auditor. This was collated and analysed by content analysis.

\section{Data management}

From 2013-2017 audit data was entered into the PIPPA Access database. In 2018 a state-wide data management system was used - QARS. Both databases enabled the generation of descriptive statistics at district, facility and ward level.

The financial costs of auditing involve both auditor training and data collection time. Additional costs include the coordinators' time in preparing and data entry in the years where the audit was paper-based. While the audit team was made up of a range of positions - registered nurse, clinical nurse specialist, clinical nurse consultant, nurse practitioner, podiatrist, occupational therapist and junior medical officer to estimate costs an average hourly rate of $A \$ 37$ was based on a registered nurse Level 4 as specified in the New South Wales Nurses and Midwifery Award Audit ${ }^{14}$.

The questionnaires' responses were coded and analysed using content analysis, and the rigour was supported using the framework of other qualitative researchers ${ }^{15}$. The 
Table 1. NSLHD facility profiles

\begin{tabular}{|c|c|c|c|}
\hline Facility & Peer group & Beds & Case mix \\
\hline H1 & $\begin{array}{l}\text { Principal referral } \\
\text { A1 }\end{array}$ & 600 & $\begin{array}{l}\text { - Emergency department } \\
\text { - } \text { Maternity } \\
\text { - } \text { ICU (60) } \\
\text { - Spinal } \\
\text { - } \text { Burns } \\
\text { - Mental health }\end{array}$ \\
\hline H2 & $\begin{array}{l}\text { District } \\
\text { C1 }\end{array}$ & 125 & $\begin{array}{ll}\text { - } & \text { Emergency department } \\
\text { - } & \text { Rehabilitation }(60) \\
\text { - } & \text { ICU/CCU }(6+6) \\
\text { - } & \text { Birth unit }\end{array}$ \\
\hline H3 & $\begin{array}{l}\text { Major hospital } \\
\text { B1 }\end{array}$ & 150 & $\begin{array}{ll}\text { - } & \text { Emergency department } \\
\text { - } & \text { Maternity } \\
\text { - } & \text { Rehabilitation }(60) \\
\text { - } & \text { ICU/HDU }(10+6) \\
\text { - } & \text { Mental health }\end{array}$ \\
\hline H4 (2013-2018) & $\begin{array}{l}\text { Major hospital } \\
\text { B2 }\end{array}$ & 90 & $\begin{array}{ll}\text { - } & \text { Emergency department } \\
\text { - } & \text { Maternity } \\
\text { - } & \text { Rehabilitation }(60) \\
\text { - } & \text { ICU/HDU }(8+4)\end{array}$ \\
\hline H4 (>2018) & Rehabilitation & 60 & $\begin{array}{l}\text { - Urgent care centre } \\
\text { - Rehabilitation (60) }\end{array}$ \\
\hline H5 (decommissioned October 2018) & $\begin{array}{l}\text { Major hospital } \\
\text { B2 }\end{array}$ & 210 & $\begin{array}{ll}\text { - } & \text { Emergency department } \\
\text { - } & \text { Maternity } \\
\text { - } & \text { ICU/HDU }(10+6) \\
\text { - } & \text { Mental health }\end{array}$ \\
\hline
\end{tabular}

critical skills appraisal checklist was used to determine whether quantitative and qualitative methods met acceptable standards for research rigour ${ }^{16}$.

\section{Results}

\section{Audit costs}

The number of auditors trained per site is provided in Table 2, and a breakdown of auditor training costs across the LHD of approximately $\mathrm{A} \$ 2000$ annually is contained in Table 3. Many sites had high auditor retention meaning that training costs were minimised. Some sites had both a high auditor and coordinator turnover. In these instances, training costs increased, and the need for mentoring time for the site clinical lead from the LHD facilitator also increased.

Table 3 details estimated auditor training costs which are based on one hour of face-to-face or on-line training time. The average cost per annum for training is $A \$ 2,272$.

Audit costs were calculated based on the number of auditors with an estimated 4 hours per auditor per site. Table 4 contains a breakdown of auditor numbers at each site.
Table 5 details estimated PIPPA costs which are based on 4 hours of audit time. The average total cost per annum to perform the audits is $A \$ 15,798$. Total audit costs over 7 years were $A \$ 110,593$ with an average of $A \$ 157,98$ pa across the five facilities. To calculate total costs of auditing, the auditor training costs were added to the audit costs; these are detailed in Table 6. The total cost to the district per annum is a conservative estimate of $A \$ 17,460$ per annum.

\section{Audit benefits}

The opportunity cost of time diverted to auditing on this audit requires expertise in skin and classification of skin injuries. However, reliability of data arises from training and skin injury classification testing to ensure that auditors accurately identify skin injury (IAD/pressure injury) and severity. This auditor training increases skills in the clinical workforce in skin assessment, leading to more accurate classification and targeted interventions at times outside of the audit. This is of significant benefit, particularly when identification is required on entry to a facility to exclude pre-existing pressure injuries from being documented and coded as a HAPI. 
Table 2. Number of auditors trained

\begin{tabular}{|c|c|c|c|c|c|c|}
\hline & H1 & H2 & H3 & H4 & H5 & Total \\
\hline 2013 & 26 & 9 & 13 & 8 & 14 & 70 \\
\hline 2014 & 10 & 0 & 0 & 6 & 1 & 17 \\
\hline 2015 & 25 & 10 & 10 & 5 & 8 & 58 \\
\hline 2016 & 47 & 24 & 0 & 3 & 4 & 78 \\
\hline 2017 & 33 & 12 & 12 & 4 & 0 & 61 \\
\hline 2018 & 47 & 15 & 19 & No audit & 5 & 86 \\
\hline 2019 & 45 & 10 & 0 & 5 & Closed & 60 \\
\hline Total & 233 & 80 & 54 & 31 & 32 & 430 \\
\hline
\end{tabular}

Table 3. Estimated auditor training costs $(A \$)$

\begin{tabular}{|l|l|l|l|l|l|l|} 
& H1 & H2 & H3 & H4 & H5 & Total \\
\hline 2013 & $\$ 962$ & $\$ 333$ & $\$ 481$ & $\$ 296$ & $\$ 518$ & $\$ 2590$ \\
\hline 2014 & $\$ 370$ & Nil trained & Nil trained & $\$ 222$ & $\$ 37$ & $\$ 629$ \\
\hline 2015 & $\$ 925$ & $\$ 370$ & $\$ 370$ & $\$ 185$ & $\$ 296$ & $\$ 2146$ \\
\hline 2016 & $\$ 1739$ & $\$ 888$ & $\$ 0$ & $\$ 111$ & $\$ 111$ & $\$ 2886$ \\
\hline 2017 & $\$ 1221$ & $\$ 444$ & $\$ 444$ & $\$ 148$ & Nil trained & $\$ 2257$ \\
\hline 2018 & $\$ 1739$ & $\$ 555$ & $\$ 703$ & No audit & $\$ 185$ & $\$ 3182$ \\
\hline 2019 & $\$ 1665$ & $\$ 370$ & Nil trained & $\$ 185$ & Closed & $\$ 2220$ \\
\hline Total & $\$ 7697$ & $\$ 2960$ & $\$ 1998$ & $\$ 1147$ & $\$ 1147$ & $\$ 15910$ \\
\hline Average & $\$ 1099$ & $\$ 422$ & $\$ 285$ & $\$ 191$ & $\$ 191$ & $\$ 2272$ \\
\hline
\end{tabular}

Table 4. Auditor numbers

\begin{tabular}{|l|l|l|l|l|l|l|} 
& H1 & H2 & H3 & H4 & H5 & Total \\
\hline 2013 & 27 & 10 & 15 & 10 & 15 & 77 \\
\hline 2014 & 30 & 14 & 12 & 8 & 8 & 72 \\
\hline 2015 & 41 & 17 & 15 & 5 & 8 & 86 \\
\hline 2016 & 79 & 21 & 21 & 11 & 9 & 141 \\
\hline 2017 & 63 & 23 & 22 & 11 & 10 & 129 \\
\hline 2018 & 72 & 19 & 26 & No audit & 5 & 122 \\
\hline 2019 & 76 & 18 & 26 & 7 & Closed & 118 \\
\hline Total & 387 & 122 & 137 & 52 & 55 & 754 \\
\hline Average pa & 55 & 17 & 20 & 7 & 8 & 107 \\
\hline
\end{tabular}


Table 5. Estimated audit costs (A\$)

\begin{tabular}{|l|l|l|l|l|l|l|} 
& H1 & H2 & H3 & H4 & H5 & Total \\
\hline 2013 & $\$ 3996$ & $\$ 1480$ & $\$ 2220$ & $\$ 1480$ & $\$ 2220$ & $\$ 11396$ \\
\hline 2014 & $\$ 4440$ & $\$ 2072$ & $\$ 1776$ & $\$ 1184$ & $\$ 1184$ & $\$ 13172$ \\
\hline 2015 & $\$ 6068$ & $\$ 2516$ & $\$ 2220$ & $\$ 740$ & $\$ 1184$ & $\$ 18796$ \\
\hline 2016 & $\$ 11692$ & $\$ 3108$ & $\$ 3108$ & $\$ 1628$ & $\$ 1332$ & $\$ 20868$ \\
\hline 2017 & $\$ 9324$ & $\$ 3404$ & $\$ 3256$ & $\$ 1628$ & $\$ 1480$ & $\$ 19092$ \\
\hline 2018 & $\$ 10656$ & $\$ 2812$ & $\$ 3848$ & No audit & $\$ 740$ & $\$ 18056$ \\
\hline 2019 & $\$ 10249$ & $\$ 2664$ & $\$ 3848$ & $\$ 1036$ & Closed & $\$ 1464$ \\
\hline Total & $\$ 56425$ & $\$ 18056$ & $\$ 20276$ & $\$ 7696$ & $\$ 8140$ & $\$ 110593$ \\
\hline Average pa & $\$ 8060$ & $\$ 2579$ & $\$ 2896$ & $\$ 1283$ & $\$ 1357$ & $\$ 15798$ \\
\hline
\end{tabular}

Table 6. Estimated total audit costs $(A \$)$

\begin{tabular}{|l|l|l|l|l|l|l|} 
& H1 & H2 & H3 & H4 & H5 & Total \\
\hline 2013 & $\$ 4958$ & $\$ 1813$ & $\$ 2701$ & $\$ 1776$ & $\$ 2738$ & $\$ 13986$ \\
\hline 2014 & $\$ 4810$ & $\$ 2072$ & $\$ 1776$ & $\$ 1406$ & $\$ 1221$ & $\$ 11285$ \\
\hline 2016 & $\$ 6993$ & $\$ 2886$ & $\$ 2590$ & $\$ 925$ & $\$ 1480$ & $\$ 14874$ \\
\hline 2017 & $\$ 13431$ & $\$ 3996$ & $\$ 3108$ & $\$ 1739$ & $\$ 1443$ & $\$ 23717$ \\
\hline 2018 & $\$ 10545$ & $\$ 3848$ & $\$ 3700$ & $\$ 1776$ & $\$ 1480$ & $\$ 21349$ \\
\hline 2019 & $\$ 12395$ & $\$ 3367$ & $\$ 4551$ & No audit & $\$ 925$ & $\$ 21238$ \\
\hline Total & $\$ 11581$ & $\$ 3219$ & $\$ 3848$ & $\$ 1221$ & Closed & $\$ 19869$ \\
\hline Average pa & $\$ 9244$ & $\$ 21201$ & $\$ 18174$ & $\$ 8843$ & $\$ 9287$ & $\$ 122218$ \\
\hline
\end{tabular}

There are a number of benefits that arise from the PIPPA. These can be attributed to the generation of data which allows monitoring of the scope of pressure injuries. This data can be trended over time within our dataset or can be used to benchmark with external peers. Another benefit is to auditors arising from training and auditing.

\section{Serial data}

The audit framework has provided accurate serial prevalence data; this is summarised in Figure 1 which graphically represents the overall pressure injury point prevalence and the HAPI point prevalence. The downward trend and the widening of the gap between all pressure injuries and HAPIs is a good outcome for the district. This data provides an indicator of quality and safety for patient care relating to pressure injury prevention which is important evidence for accreditation. Good performance flows on to reduced length of stay, resource utilisation and costs, as well as enhanced patient satisfaction.

\section{Auditor benefits}

After each audit, auditors were given the opportunity to provide feedback via a questionnaire. Table 7 summarises the benefits highlighted by auditors. Auditors generally viewed the audit as an opportunity for team-building, raising awareness, and identifying the barriers to pressure injury prevention and management. This awareness and upskilling resulted in an understanding of deficiencies and a link to improving the reporting, documentation and interventions relating to pressure injury prevention.

\section{Discussion}

The high cost of PIPPA has not been highlighted previously in the Australian setting. Our estimation of the financial and opportunity costs of PIPPA has provided food for thought about the cost versus benefit of this process. While there are difficulties in relying on medical record coded data for estimating the size of the problem, PIPPA offers an option 
Figure 1. Serial point prevalence and HAPI point prevalence

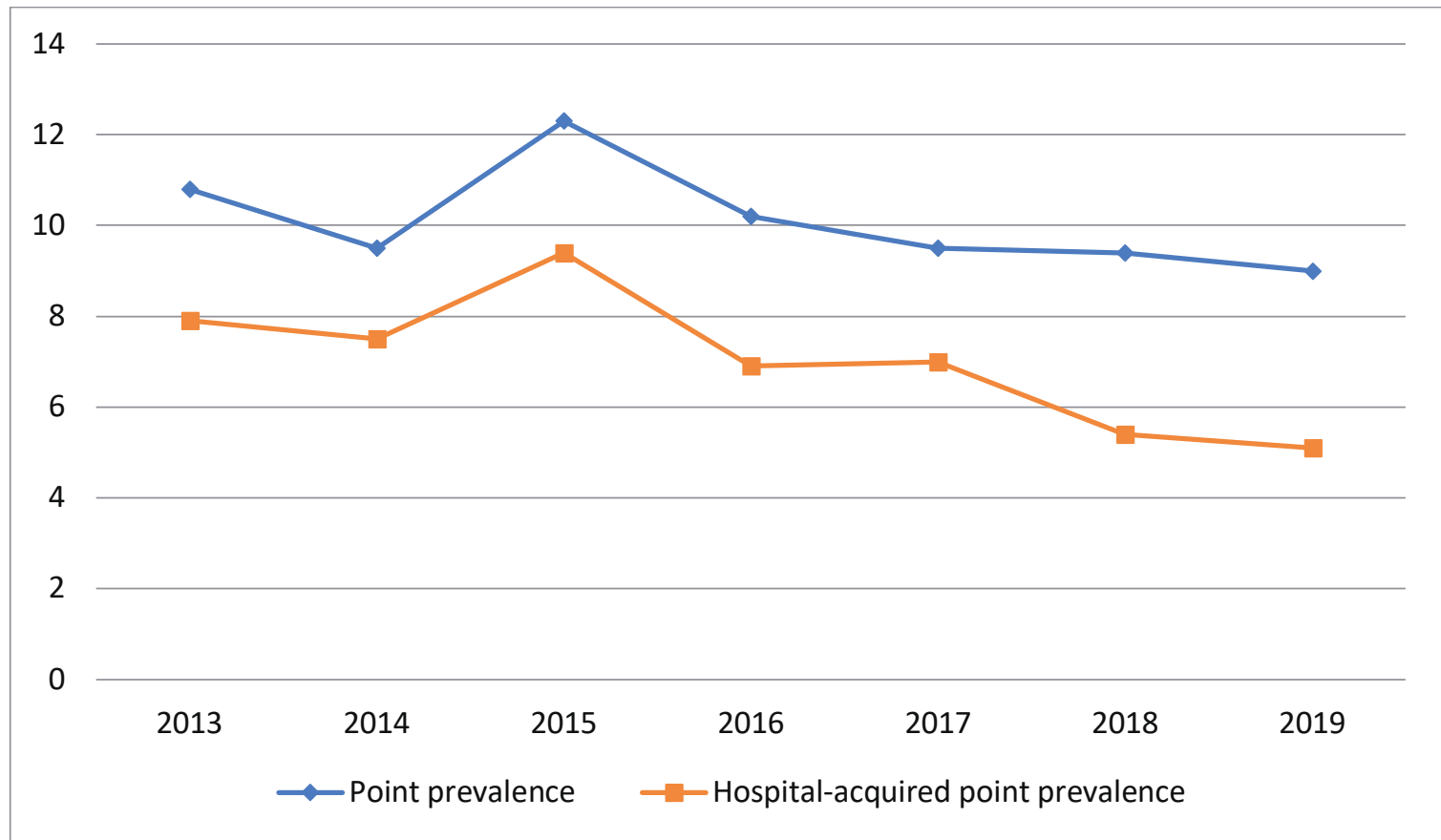

for alternative data on pressure injuries. However, this is at a high cost to the health service. There is an opportunity cost of clinician time diverted to auditing as this audit requires expertise in skin assessment and classification of skin injuries. However, the reliability of data arises from training and testing to ensure that auditors accurately identify pressure injuries and grade severity. The option of online training used in conjunction with face-to-face education may offer some cost savings but, in our district, this has not been taken up due to the preference to localise training to each facility and also ensure accurate skin assessment and thorough competency testing in the classification of skin injuries.

In addition, the implementation of the electronic medical has resulted in difficulty both recording and locating documentation about skin and pressure injury prevention. After implementation, the auditor training required content on locating information in the electronic medical record, for example the use of a filter for searching and navigation in the document launcher. This was challenging for some of the auditors and may have resulted in missed data capture about compliance with other items measured in the audit, for example mandatory reporting. The electronic record did, however, assist in locating documentation once training which improved the search strategies of the auditors.

One of the benefits of the program has been clinician engagement with patient safety and quality, and the opportunity to get involvement in closing the quality improvement loop. There have also been incidental learning opportunities which both raise awareness and model best practice in numerous areas including skin assessment, pressure injury risk assessment, care planning and documentation. In addition, the PIPPA has provided opportunities to enhance the transition from paper to electronic medical record with the auditors being up-skilled in navigating the electronic medical record through auditor training.

Some of the limitations of the PIPPA framework arise as a result of poor retention of the lead clinician at each site as well as trained auditors. Auditing a part of a district-wide program requires consistency and local clinical leadership. Retention of the clinical lead at a number of sites challenged the operationalisation of the audits. These sites required more auditor recruitment, resulting in increased costs of audits through auditor training. They lost time efficiencies which result from experienced auditors who can reduce audit time. Other issues have included the transition from the Access database to the state-wide point of audit data management system which required further training and data handling to provide reliable results. For example, audit tool deficiency, compared to an established data management framework refined by WoundsWest, has resulted in the loss of capacity to perform wound prevalence audits. Another change which complicated the program included the introduction of a new pressure injury staging system (2014) necessitating an adjustment to the database and difficulty making comparisons from year to year.

\section{Limitations}

The calculation of audit costs can only be considered approximate as the average hourly rate of a registered nurse Level 4 was selected in an attempt to average out the wide variability in audit team composition. 
Table 7. Summary of auditor feedback - benefits

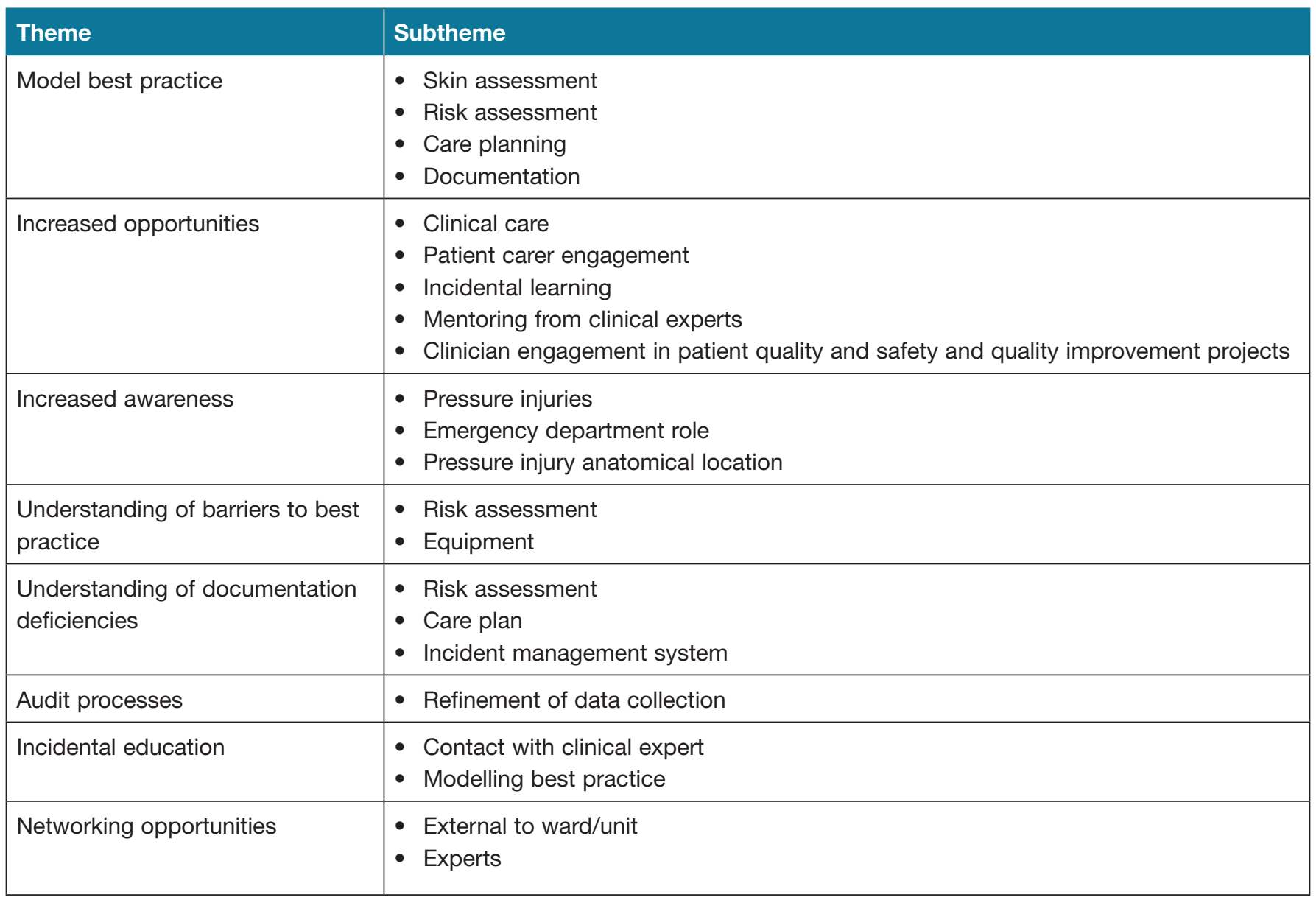

\section{Conclusion}

The state-wide pressure injury policy, which mandated the annual PIPPA, has had far-reaching impacts on the clinical workforce who were largely responsible for data collection and management in our district. By adopting a rigorous PIPPA methodology, the value of what began as a quality improvement activity became one dataset of a mixed methods study to evaluate a new model of care. The benefits included increased awareness of pressure injuries and auditor understanding of the barriers to strategy implementation and documentation. The auditing program occurred when there was a significant change in clinical practice (for example new practice guidelines) and documentation (for example the electronic medical record). However, whilst the robust auditing framework resulted in inaccurate data capture, there is a need to acknowledge the operational costs (training and audit) and the opportunity costs (lost clinical time) which now require a revaluation of the need for this as an ongoing annual audit.

\section{Ethics}

PIPPA is one dataset of a mixed methods study for measuring the implementation of a skin and wound integrated model of care (SWIM). The SWIM research project was approved by the Ethics Committee of the NSLHD. PIPPAs were conducted in accordance with this ethical approval and using audit methodology endorsed by the NSW Clinical Excellence Commission.

\section{Conflict of interest}

The authors have no conflicts of interest to report.

\section{Funding}

The authors received no funding for this study.

\section{References}

1. Graves $\mathrm{N}$, Zheng $\mathrm{H}$. Modelling the direct health care costs of chronic wounds in Australia. Wound Practice Res 2014;1:20-33.

2. Nguyen $\mathrm{K}-\mathrm{H}$, Chaboyer W, Whitty JA. Pressure injury in Australian public hospitals: a cost-of-illness study. Aust Health Rev 2015;39(3):329-36.

3. Australian Commission on Safety and Quality in Health Care. National standards and quality health service standards; 2012.

4. Australian Commission on Safety and Quality in Health Care. National Safety and Quality Health Service Standards. 2nd ed. Sydney: ACSQHC; 2017. 
5. AWMA. AWMA Panpacific pressure injury prevention and management guidelines. 2012.

6. National Pressure Ulcer Advisory Panel, European Pressure Ulcer Advisory Panel, Pan Pacific Pressure Injury Alliance. Prevention and treatment of pressure ulcers. Osborne Park, WA: Cambridge Media; 2014.

7. European Pressure Ulcer Advisory Panel (EPUAP), National Pressure Injury Advisory Panel (NPIAP), Pan Pacific Pressure Injury Alliance (PPPIA). Prevention and treatment of pressure ulcers/injuries: clinical practice guidelines. The international guideline. 3rd ed. Emily Haesler, editor. EPUAP, NPIAP, PPPIA; 2019.

8. NSLHD. PO2008_038 Pressure injury prevention program policy. In: CGU, editor. 2013.

9. NSW Ministry of Health. PD2014_007 Pressure injury prevention and management. In: CEC, editor. 2014.

10. Australian Commission on Safety and Quality in Health Care. Hospital acquired complications toolkit. Sydney 2018.

11. Prentice J, Stacey M, Lewin G. An Australian model for conducting pressure ulcer prevalence surveys. Primary Intent 2003;11(2):87-8,90-1,3-6,8-100,2-9.

12. Prentice J, Strachan V, Carville K, Santamaria N, Elmes R, Della P. WoundsWest: delivering comprehensive strategies to improve wound management in Western Australian Health Services. Wound Practice Res 2009;17(3):122.

13. Tubaishat A, Papanikolaou P, Anthony D, Habiballah L. Pressure ulcer prevalence in acute care settings: a systematic review 2000-2015. Clinical Nurs Res 2018;27(6):16.

14. Industrial Relations Commission of NSW. Public Health System Nurses' and Midwives' (State) Award 2019. 2019.

15. Graneheim UH, Lindgren BM, Lundman B. Methodological challenges in qualitative content analysis: a discussion paper. Nurse Ed Today 2017;56:29-34.

16. The Oxford Centre for Triple Value Healthcare. Critical Skills Appraisal Programme Checklists [website]. Oxford; 2014 Available from: http://www.casp-uk.net/casp-tools-checklists 